\title{
Life quantity, life quality and longevity: An intertemporal social evaluation framework
}

\author{
Jean-Yves Duclos
}

\section{Bouba Housseini}

C Jean-Yves Duclos, Senior Fellow at Ferdi, is the Director and a full Professor at the Department of economics at Laval University. He is also member of CIRPEE Centre interuniversitaire sur le risque, les politiques économiques et l'emploi.

Email: jyves@ecn.ulaval.ca

\section{Bouba Housserni, Universit'e Laval and CIRP'EE \\ Email: bouba.housseini.1@ulaval.ca}

\section{Abstract}

The evaluation of development processes and of public policies often involves comparisons of social states in which populations differ in size and longevity. This requires social evaluation principles to be sensitive to both the number and the length of lives. This paper explores the use of axiomatic and welfarist principles to assess social welfare in that framework. It attempts to overcome some of the limits of existing methods in the literature, in particular by avoiding a temporal repugnant conclusion, by neither penalizing nor favoring life fragmentation, and by satisfying critical-level temporal consistency. It does this by characterizing a critical-level lifetime utility function that values life periodically. To address some of the controversies on discounting utilities across time, two alternative versions of the function are developed, one with discounting and one without.

JEL Classification: C02, D31, D63, I31, J17.

Keywords: Intertemporal social evaluation, population ethics, critical-level utilitarianism, lifetime utility, social discounting.

\section{Acknowledgments}

This work was carried out with support from the William and Flora Hewlett Foundation through a dissertation fellowship administrated by the Institute of International Education (New York, NY). We are grateful to John Cockburn and participants at the 11th biannual meeting of the SSCW and at the 2011 SCSE annual conference for useful comments and advice. 


\section{Introduction}

\subsection{Context}

All around the world, development is associated with improved longevity and slower demographic growth. Global average life expectancy at birth has risen from 63 years in 1980 to 70 years in 2011, while the global annual population growth rate has fallen from $1.8 \%$ to $1.2 \%$ during the same period. Developed countries have already crossed what is commonly called the critical point of a "demographic transition." Such a critical point is characterized by relatively high longevity and by relatively low fertility. This demographic transition is also starting to occur in the developing world. Do these economic, health and demographic changes improve the "overall value of our societies," i.e., their social welfare? The answer would at first sight seem to be an obvious "yes."

Upon second thought, however, one may note that these demographic changes may lead to social and economic pressures on labour markets and on social security systems (to take two examples), and that some of these pressures may inflict costs on society. There may also be a limit to which falling fertility rates may be sustainable from the perspective of long-term sustained economic development.

Perhaps more importantly, from this paper's normative perspective, is the question of the extent to which these changes actually increase social welfare. Answering this question is fundamental to assessing differences in social welfare over time and across societies. It is also useful for addressing the trade-off that may exist between changes in the quantity (as measured by longevity and population size) and in the quality (individual welfare in each period) of welfare along the process of development. Despite the importance of the topic, the literature has largely ignored that trade-off. Social welfare is traditionally evaluated in a timeless framework, based solely on quality of life (usually captured by living standards) and ignoring the quantity of it (longevity and population size). The main objective of this paper is thus to explore the normative elements of this trade-off by setting up a social evaluation framework that can be used to account jointly for population size, longevity and the distribution of periodic welfare.

\subsection{Literature}

Blackorby et al. (1995, 1996a,b, 1997a,b) provide seminal contributions to intertemporal social evaluation. They consider two types of social evaluation functions (SEF): those based on Classical Generalized Utilitarianism (CGU) and those based on Critical Level Generalized Utilitarianism (CLGU). CGU defines social welfare as a double sum of transformed periodic utilities across individuals and time. The problem with classical utilitarianism is that it is subject to a repugnant conclusion (Parfit, 1984): with classical utilitarianism, a sufficiently large population will necessarily be deemed better than any 
other smaller population, even when the larger population has very low average utility. CLGU does not have this flaw since it grants positive contributions to social welfare only for those whose lives are "worth living." Its value function is a sum of lifetime utilities net of the critical level, defined as the level of lifetime utility for an additional individual that does not affect the social utility function (Blackorby and Donaldson, 1984). It can be viewed as a sort of ethical threshold for valuing lives in an intertemporal framework.

However, the intertemporal CLGU principles used by Blackorby et al. (1995, 1997a) introduce another type of repugnant conclusion. An ordering of two individual lifetime utilities is subject to a temporal repugnant conclusion if it can always evaluate the life of a sufficiently long-life individual who is destitute in every period to have been better than that of any richer individual with a shorter life. This conclusion may indeed be deemed to be repugnant if one feels that longevity is welfare increasing only for those additional periods that are worth living: ceteris paribus, an increase in longevity should not always increase social welfare.

This view may also be set in the context of Dalgaard and Strulik (2011), who analyze the optimal timing of death. Dalgaard and Strulik (2011) develop a model of intertemporal decision making in which the speed of the aging process - and consequently the time of death — is endogenously determined by optimal health investments, in part to explain the Preston curve (e.g. Preston, 1975) of a positive relationship between income and life expectancy. The earlier literature on optimal health investments (Ehrlich and Chuma, 1990; Grossman, 1972, among others) also generally provides support to the idea that it is not always desirable, from an individual perspective, to extend a life beyond a certain length, given that the quality and the length of life may be substitutable in the value of welfare. The paper extends those arguments to a social evaluation context.

The intertemporal CLGU functions developed by Blackorby et al. (1995, 1997a) further exhibit a preference for "unfragmented lives". A preference for unfragmented lives penalizes population renewal and may encourage some awkward demographic features in the long run.

\subsection{Contribution of paper}

In order to satisfy critical-level temporal consistency and indifference towards fragmentation of lives, and to avoid a temporal repugnant conclusion, this paper adopts a critical-level procedure applied periodically rather than over a lifetime. This leads to the characterization of a critical-level lifetime utility function that values utilities periodically. We develop two different versions of the function: with and without discounting.

A social evaluation framework that is jointly sensitive to both longevity and economic growth nicely complements recent changes in the human development evaluation paradigm. Development objectives have indeed evolved significantly in recent decades, shifting partly 
away from traditional income growth objectives towards broader human development ones. Longevity and health are prominent objectives among these, as illustrated below in the first UNDP Human Development Report:

"Human development is a process of enlarging people's choices. In principle, these choices can be infinite and change over time. But at all levels of development, the three essential ones are for people to lead a long and healthy life, to acquire knowledge and to have access to resources needed for a decent standard of living. If these essential choices are not available, many other opportunities remain inaccessible" (UNDP, 1990, p. 10.).

UNDP's stance has been influential in spurring a multidimensional approach to social evaluation. What has not been recognized, however, is that there may be trade-offs between standards of living, longevity and population size. Our proposed intertemporal social evaluation framework provides such an explicit trade-off.

The rest of the paper is organized as follows. Section 2 develops an undiscounted intertemporal social evaluation function. We first set up the methodological framework and then present and challenge some of the features of existing intertemporal SEFs. We then develop principles of intertemporal social evaluation and axiomatically characterize the corresponding value function. Section 3 develops the discounted version of our framework. We first introduce the discounting principle and link the discounted and undiscounted frameworks. We then characterize a discounted SEF and a geometrically-discounted one. Section 4 concludes the paper. Most of the proofs appear in the appendix.

\section{Intertemporal social evaluation without discounting}

\subsection{Notation and methodological framework}

This paper is based on the same analytical framework as Blackorby et al. (1996a). Our approach is welfarist, in that it only uses utilities enjoyed by individuals during the period of evaluation. A social state $x \in \mathcal{X}$ is defined by a matrix of utilities $M \in \mathcal{M}$ that gives utilities for each individual in each period. We define $M=\left\{u_{i, j}\right\}_{i \in \mathcal{N} ; j \in \mathcal{T}}$, where

$\mathcal{N}=\{1,2, \ldots, n\}$ is the set of individuals and $\mathcal{T}=\{1,2, \ldots, T\}$ is the time frame being evaluated. Thus, the total number of individuals is $n$ and the last period of evaluation is $T$. The periods can be days, months, years, etc.

Individuals have different dates of birth and different lengths of life. Period 1 corresponds to the date of birth of the first individuals born and period $T$ is the date of death of the last persons alive. Thus, during the period of evaluation, we could have some individuals not being born yet at the beginning and other ones who die before $T$. For individuals 
not alive during some periods, the corresponding utilities are blank in the utility matrix $M$. An example of such a matrix $M$ is:

\begin{tabular}{|c|c|c|c|c|c|c|c|}
\hline Persons $\backslash$ periods & 1 & 2 & 3 &. &. & $T-1$ & $T$ \\
\hline 1 & & $u_{1,2}$ & $u_{1,3}$ &.. &.. & $u_{1, T-1}$ & \\
\hline 2 & $u_{2,1}$ & $u_{2,2}$ & $u_{2,3}$ & & & & \\
\hline 3 & & & & $u_{3, .}$ & $u_{3, .}$ & $u_{3, T-1}$ & $u_{3, T}$ \\
\hline$\cdot$ & $u_{., 1}$ & $u_{., 2}$ & $u_{., 3}$ & $u_{., .}$ & $u_{., .}$ & & \\
\hline$\cdot$ & & $u_{., 2}$ & $u_{., 3}$ & & & & \\
\hline$n$ & $u_{n, 1}$ & $u_{n, 2}$ & $u_{n, 3}$ &.. &.. & $u_{n, T-1}$ & $u_{n, T}$ \\
\hline
\end{tabular}

Our main goal here is to define a social ordering $R$ that can be used to rank the different elements of $\mathcal{M}$. In the process, we would like to characterize a well-defined social evaluation function $W^{n, T}$, representing $R$.

\subsection{Principles of intertemporal social evaluation}

A natural starting point adopts a two-step aggregation approach, first, to generate a lifetime utility function $V$ representing an individual ordering $R_{I}$, and, second, to aggregate the vectors of lifetime utilities and obtain the social evaluation function $W^{n, T}$. This approach is widespread in the literature: Blackorby et al. (1995, 1996a, 1997a,b); Maasoumi and Zandvakili (1986); Salas and Rabadan (1998). It leads to the following intertemporal classical generalized utilitarian function:

$$
W(u)=\sum_{i \in \mathcal{N}} \sum_{t \in \mathcal{T}} g\left(u_{i t}\right)
$$

Blackorby et al. (1996a) justify this through two different temporal consistency axioms, the full temporal consistency and the forward looking consistency axioms. The full temporal consistency axiom states that social evaluation should not be affected by the order of aggregation of the matrix of utilities (lines then columns or columns then lines), under certain conditions. This axiom requires the ranking of two utility matrixes, which differ only by their $t^{\text {th }}$ column of utilities, to be the same regardless of whether they are ordered using a regular intertemporal social ordering $R$ or a social ordering $R_{t}$ in each period that only considers the welfare information in that same period $t$. Blackorby et al. (1996a) show that if this axiom is combined with traditional welfarist axioms - the strong Pareto, continuity and anonymity axioms — the resulting social evaluation function fits within Classical Generalized Utilitarianism. Their forward looking consistency axiom leads to the same result. The forward looking consistency axiom requires the ranking of any two states that differ only from period $t$ to be the same under the regular social ordering 
$R$ and a forward-looking social ordering $R_{t}^{+}$that considers only the temporal information from period $t$ onwards.

Unfortunately, Classical Generalized Utilitarianism is subject to a so-called "repugnant conclusion". The fact that classical (or total) utilitarianism may suffer from a "repugnant conclusion" is now widely known in welfare economics and has been popularized by Parfit (1984):

"For any possible population of at least ten billion people, all with a very high quality of life, there must be some much larger imaginable population whose existence, if other things are equal, would be better, even though its members have lives that are barely worth living" (Parfit, 1984, p.388).

To avoid Parfit's repugnant conclusion, a timeless CLGU principle is developed in Blackorby and Donaldson (1984). Its intertemporal version is characterized in Blackorby et al. (1995) using the traditional welfarist axioms (more on this below) and an axiom called Independence of the Utility of the Dead (IUD). ${ }^{1}$ Unlike Blackorby et al. (1996a) who use a two-dimensional matrix of utilities, the intertemporal CLGU function considers a single vector of lifetime utilities. Blackorby et al. (1996a) also invoke an individual welfarism axiom to make lifetime utility equal to a sum of periodic utilities. According to this, all positive utilities are necessarily welfare increasing at the individual level; a person prefers to live an additional unit of life as long as the utility in this additional period is positive. The CLGU value function is then:

$$
W(u)=\sum_{i \in \mathcal{N}}\left[g\left(u_{i}\right)-g(\alpha)\right]
$$

where $u_{i}$ is the lifetime utility of individual $i$ (assumed to be the sum of periodic utilities) and $\alpha$ is the critical level.

The additive formulation is a consequence of the "Independence of the Utility of the Dead" axiom. Removing past utilities which are common to two given alternatives must not change their social ranking. The axiom seems particularly useful when comparing two social states with a common past:

"Suppose that, in any two alternatives, the same individuals are born and die before period $t$ and have the same birthdates, lengths of life, and lifetime utilities. Independence of the Utility of the Dead requires that, if these individuals are removed from the alternatives in question, the social ranking is unchanged"(Blackorby et al., 1995).

\footnotetext{
${ }^{1}$ This is referred to later in Blackorby et al. (2005) as the "Independence of the Existence of the Dead" axiom.
} 
Only the utilities of those individuals living at least one period beyond the present may enter the social evaluation exercise. Unfortunately, this specification of the CLGU principle exhibits some undesirable features that we discuss in the following subsection.

\subsection{Alternative principles for intertemporal social evaluation}

This section identifies additional proprieties that the individual ordering $R_{I}$ and the social ordering $R$ may have to satisfy and their implications for the specification of individual utility and social evaluation functions. The objective is to extend the literature's existing methods based on some incompatibility between existing functions and some desirable social evaluation principles as well as to provide alternative social evaluation functions. Identifying the set of all candidate value functions may also be useful, such as when performing robustness checks and dominance tests.

The above discussion suggests a temporal formulation of the repugnant conclusion.

\section{Definition 1. (Temporal repugnant conclusion)}

An individual ordering $R_{I}$ is subject to a temporal repugnant conclusion if it can always rank a poor individual with a sufficiently long life as necessarily better off than a richer one whose length of life is shorter.

The temporal repugnant conclusion arises whenever an individual $i$ is deemed better off than another individual $j$ simply because his life is sufficiently longer than that of $j$, regardless of how low his average utility may be. It may instead be believed that longevity is not always welfare increasing, and that it may be so only for those additional periods worth living.

\section{Critical-level temporal consistency}

A tension can also exist between periodic welfarism and application of the critical level principle over the course of a lifetime. Periodic assessments of welfare may indicate that a life is worth living during every period of the life (with utilities at every period sufficiently above the periodic critical level), even in cases where the application of a lifetime critical level (applied over the distribution of lifetime utilities) indicates that the life, considered as a whole, may not be worth living if lifetime utility is below the lifetime critical level.

To see this better, let $g(u)=u$ and consider a person with positive utilities $u_{i t} \geq \alpha, T_{i}$ being his life duration and $\bar{T} \alpha$ being some fixed lifetime critical level. Periodic welfarism stipulates that every period of this person's life is worth living, whereas the lifetime criticallevel principle may lead to the conclusion that his life, considered as a whole, is not worth living (given that his lifetime utility $\sum_{t} u_{i t}$ may be below $\bar{T} \alpha$ but above $T_{i} \alpha$ ). A set of consistent periodic and temporal principles should arguably be used. 


\section{Definition 2. (Critical-level temporal consistency)}

A social ordering $R$ and an individual ordering $R_{I}$ satisfy critical-level temporal consistency if their intertemporal evaluation of a life is consistent from both periodic and intertemporal perspectives.

This means that a life with periods deemed as worth living must be deemed as worth living from an intertemporal perspective as well. Although we believe that this is a reasonable property, it will not be used to help characterize the results below. The property is, however, consistent with these results.

\section{Indifference towards fragmentation of lives}

"Live simply so that others may simply live." This assertion by the famous Indian leader Gandhi alludes to the trade-off that may exist between individual quality of life, longevity and population size. One interpretation is that one should be willing to sacrifice one's own wellbeing for the benefit of others. Another is that sacrifices in living standards should be made in order to allow for the existence of others. Yet one more interpretation is that sacrifices in longevity should also be envisaged in order for others to enjoy a life too. This last interpretation, however, stands in opposition to the principle of favoring unfragmented lives that characterizes the function developed in Blackorby et al. (1995). Later, in 2005, the authors state that:

"Preventing someone's death is more important than bringing about new lives when the consequences for total utility are the same" (Blackorby et al., 2005, p.153).

This means that, as long as total utility remains unchanged, one strictly prefers a situation $x$ in which one person lives 100 years rather than a situation $y$ in which this person lives 50 years and is subsequently replaced by another person who lives the remaining 50 years, with utilities equal to those of the person in alternative $x$. Moreover:

"If a population principle's same-number sub-principles are utilitarian, a positive critical level has the effect of giving weight to unfragmented lives" (Blackorby et al., 2005, p.151).

As shown below, a population principle whose same-number sub-principles are utilitarian may nevertheless exhibit indifference towards the fragmentation of lives, even with a positive critical level. Moreover, from a normative perspective, giving weight to unfragmented lives may be inconsistent with aversion to inequality, as acknowledged by Blackorby et al. (2005): 
"If a principle has same-number sub-principles that are inequality averse generalized utilitarian, weight may not be given to unfragmented lives even if critical levels are positive" (Blackorby et al., 2005, p.152).

One solution is to position social evaluation functions on the frontier between a preference for longevity (through a preference for unfragmented lives) and a preference for population renewal (through aversion to inequality of lifetime utilities).

\section{Definition 3. (Indifference towards fragmentation of lives)}

Let $x$ be a social state of $n$ individuals with a matrix of utilities $\left(\left\{u_{i t}\right\}_{i \in \mathcal{N} ; t \in \mathcal{T}}\right)$, and let $\widehat{x}$ be another social state obtained from $x$ by replacing an arbitrary person $j$ in an arbitrary period $l$ with a new person $k$ who has the same utilities in the remaining periods as those of person $j$ in $x$. Thus, the utility matrix $\widehat{x}$ is as follows: for all $i \neq k, j, \widehat{u}_{i, t}=u_{i, t}$ and for $t \geq l, \widehat{u}_{k, t}=u_{j, t} . A$ social ordering $R$ satisfies indifference towards fragmentation of lives if it ranks $x$ and $\widehat{x}$ as equally good.

Example 1. Consider a social state $x$ defined by the utility matrix $M_{x}$ in Figure 1. Two individuals are alive in $x$ and have a total utility of 3. Let $y$ be another social state identical to $x$ except that person 1 in $x$ is replaced in the second period by a new person with the same utilities over the remaining periods. The total utility of the population remains unchanged while the total population increases from two to three persons.

Figure 1: Illustrative utility matrix: Indifference towards fragmentation of lives
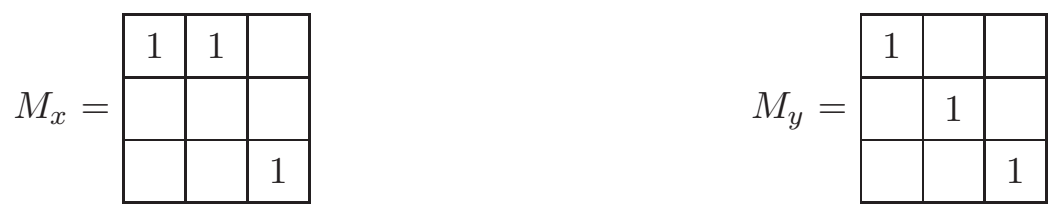

Indifference towards fragmentation of lives states that $x$ and $y$ are equally good from a social perspective; favoring unfragmented lives implies that $x$ is socially better than $y$.

\subsection{The periodic CLGU social evaluation function in a non-discounted framework}

To satisfy critical-level temporal consistency, indifference towards fragmentation of lives, and to avoid the temporal repugnant conclusion, a critical-level procedure set in each period rather than over the lifetime is now proposed. This leads to the characterization of a critical-level individual lifetime utility function that values life periodically. 


\subsubsection{A critical-level lifetime utility function}

To set up a critical-level lifetime utility function, we need a suitable intertemporal definition of the critical level before characterizing a critical-level individual ordering $R_{I}$.

Definition 4. For an individual $i$ born in $s_{i}$ and living $T_{i}$ periods, the periodic criticallevel $\alpha_{s_{i}+T_{i}}$ is the level of periodic utility that does not affect the social welfare function when enjoyed by person $i$ in an additional period of life. Thus, an additional period of life is welfare increasing if the new utility is above $\alpha_{s_{i}+T_{i}}$, and conversely it is welfare decreasing if the new utility is below $\alpha_{s_{i}+T_{i}}$.

Let $u_{i}$ be the utility vector of an individual $i$ who is born in $s_{i}$ and dies in $s_{i}+T_{i}$ : $u_{i}=\left(u_{i, s_{i+1}}, u_{i, s_{i+2}}, \ldots, u_{s_{i}+T_{i}}\right)$. The periodic critical level is defined such that this person is indifferent to the extension of his life by one period in which he would enjoy a utility level of $\alpha_{s_{i}+T_{i}}$. This means that $\left(u_{i, s_{i+1}}, u_{i, s_{i+2}}, \ldots, u_{s_{i}+T_{i}}\right)$ I $\left(u_{i, s_{i+1}}, u_{i, s_{i+2}}, \ldots, u_{s_{i}+T_{i}}, \alpha_{s_{i}+T_{i}}\right)$, where $I$ denotes the indifference ordering. This statement is reminiscent of the popular saying that: "Life is not measured by the number of breaths we take but by the moments that take our breath away" (anonymous quote featured in Schultz 2003's bestseller travel book). Longevity (or the number of breaths) itself does not matter as such, except for those good moments lived periodically. From this perspective, the critical level subdivides moments in one's life into two categories: those that "take our breath away" (sufficiently so!) and those that do not.

We move towards defining an individual ordering $R_{I}$ by positing the individual version of the three usual welfarism axioms: strong Pareto, anonymity and continuity.

Definition 5. (Individual strong Pareto) For any individuals $i$ and $j$, respectively with utility vectors $\left(\left\{u_{i, s_{i}+t}\right\}_{1 \leq t \leq T_{i}}\right)$ and $\left(\left\{u_{j, s_{j}+t}\right\}_{1 \leq t \leq T_{j}}\right)$ where $s_{i}$ and $s_{j}$ are the birthdates of $i$ and $j$ and $T_{i}=T_{j}$ is their length of life,

1. If, for all $t \in\left\{1,2, \ldots, T_{i}\right\}, u_{i, s_{i}+t}=u_{j, s_{j}+t}$, then $i$ and $j$ are equally well off.

2. If, for all $t \in\left\{1,2, \ldots, T_{i}\right\}, u_{i, s_{i}+t} \geq u_{j, s_{j}+t}$, with at least one strict inequality, then individual $i$ is better off than individual $j$.

The individual strong Pareto axiom implies individual Pareto indifference, which is defined by point 1 in definition 5 above. The individual Pareto indifference axiom requires individual rankings to depend only on the periodic utilities of individuals. As long as the periodic utilities of two individuals with the same length of life are equal, the evaluation of their lifetime welfare must also be the same.

\section{Definition 6. (Temporal anonymity)}

Consider an individual $i$ with utility vector $\left(\left\{u_{i, t}\right\}_{t \in \mathcal{T}_{i}}\right)$ and another individual $j$ whose utility vector is obtained by a permutation of elements of $\left(\left\{u_{i, t}\right\}_{t \in \mathcal{T}_{i}}\right)$. The utility vector of 
$j$ is thus given by $u_{j, t}=u_{i, \rho(t)}$ for all $t$ where $\rho: \mathcal{T}_{i} \longmapsto \mathcal{T}_{i}$ is a permutation of elements of $\mathcal{T}_{i}$. The individual ordering $R_{I}$ satisfies temporal anonymity if it ranks $i$ and $j$ as equally well off.

This axiom is a consequence of the absence of discounting in an intertemporal social evaluation. The evaluation periods are equally weighted and the resulting ordering $R_{I}$ is not affected by the timing of utilities.

We also assume that the individual ordering $R_{I}$ satisfies continuity, which ensures the existence of a continuous value function representing the ordering $R_{I}$ (Debreu, 1959). Relaxing the assumption of continuity may lead to situations in which small changes in periodic utilities can cause large changes in lifetime welfare; a feature that is plausibly undesirable.

\section{Definition 7. (Continuity of $R_{I}$ )}

For all $\tilde{\mathcal{T}} \neq \varnothing$, the restriction on the ordering $R_{I}$ to $\{i \in \mathcal{I} \mid \mathcal{T}=\tilde{\mathcal{T}}\}$ is continuous in periodic utilities, where $\mathcal{I}$ is the set of individuals (both existing and future persons), and $\mathcal{T}$ the number of periods individuals are alive for.

Defining $i_{\varnothing}$ as a non-existing person (the null alternative), and assuming that $\alpha_{\varnothing}$ exists, there is indifference between a society with nobody alive and a society where one individual lives one period with a utility of $\alpha_{\varnothing}$. Following the same argument as in Blackorby et al. (1995), and given continuity of $R_{I}$, the non existence of $\alpha_{\varnothing}$ would imply that a society with one person alive is always better than a society with nobody alive, no matter how miserable this person might be, or conversely, it would imply that a society with one person is always worse off than a society with nobody alive, no matter how well off this person is. These two eventualities are ruled out by assuming the existence of $\alpha_{\varnothing}$.

The individual strong Pareto, temporal anonymity and continuity axioms lead to a lifetime utility function representing the ordering $R_{I}$. The lifetime utility function denoted by $V$ is defined on the set of periodic utility vectors $\mathcal{U}$ :

$$
\begin{aligned}
V: & \mathcal{U} \longrightarrow \mathbf{R} \\
& u_{i} \longrightarrow V\left(u_{i}\right)=V\left(\left\{u_{i t}\right\}_{t \in \mathcal{T}_{i}}\right) .
\end{aligned}
$$

\section{Definition 8. (Equally-Distributed Equivalent Utility)}

Let $\left\{u_{i t}\right\}_{t \in \mathcal{T}_{i}}$ be the utility vector of person $i$. The equally-distributed equivalent utility (EDEU) $\bar{u}_{i}$ is defined as the level of utility that, if enjoyed by person $i$ at every period of his life, makes person $i$ indifferent to $\left\{u_{i t}\right\}_{t \in \mathcal{T}_{i}}$, his actual utility vector.

Thus, $V\left(\bar{u}_{i} \mathbf{1}_{T_{i}}\right)=V\left(\left\{u_{i t}\right\}_{t \in \mathcal{T}_{i}}\right)$, where $\mathbf{1}_{T_{i}}$ is a vector of dimension $T_{i}$ whose elements are all equal to 1 . The continuity and increasingness of $V$ ensure the existence of the 
EDEU, which can be defined by:

$$
\bar{u}_{i}=\Upsilon^{T_{i}}\left(\left\{u_{i t}\right\}_{t \in \mathcal{T}_{i}}\right) .
$$

The rank of individual $i$ is thus entirely determined by $\left(T_{i}, \bar{u}_{i}\right)$ and we obtain:

$$
V\left(\left\{u_{i t}\right\}_{t \in \mathcal{T}}\right)=\bar{V}\left(T_{i}, \bar{u}_{i}\right)=\bar{V}\left(T_{i}, \Upsilon^{T_{i}}\left(\left\{u_{i t}\right\}_{t \in \mathcal{T}_{i}}\right)\right)
$$

The periodic critical-level principle implies that

$$
\bar{V}\left(T_{i}+1, \Upsilon^{T_{i}+1}\left(\left\{u_{i t}\right\}_{t \in \mathcal{T}}, \alpha_{s_{i}+T_{i}}\right)\right)=\bar{V}\left(T_{i}, \Upsilon^{T_{i}}\left(\left\{u_{i t}\right\}_{t \in \mathcal{T}}\right)\right)
$$

where $\alpha_{s_{i}+T_{i}}$ is the periodic critical-level of individual $i$.

In addition to these axioms, we use a temporal independence axiom similar to the axiom of independence of the utility of unconcerned individuals (for timeless social ranking). This will generate additive separability of the lifetime utility function representing $R_{I}$.

\section{Definition 9. (Independence of the utility of unconcerned periods (IUUP))}

For all $\mathcal{P} \subseteq\{1,2, \ldots, T\}$, and for all utility vectors $u_{i}, u_{j} \in \Re^{T}$ and $u_{i^{\prime}}, u_{j^{\prime}} \in \Re^{T^{\prime}}$, with $T^{\prime}=|\mathcal{P}|$, if $u_{i t}=u_{j t}$ for all $t \in\{1,2, \ldots, T\} \backslash \mathcal{P}$, and $u_{i l}=u_{i^{\prime} l}$ and $u_{j l}=u_{j^{\prime} l}$ for all $l \in \mathcal{P}$, then $u_{i} R_{I} u_{j} \Leftrightarrow u_{i^{\prime}} R_{I} u_{j^{\prime}}$.

Example 2. Consider two individuals $i$ and $j$ with utility vectors $u_{i}$ and $u_{j}$. Let $i^{\prime}$ and $j^{\prime}$ be two other individuals whose utility vectors are obtained respectively from $u_{i}$ and $u_{j}$ by removing the two last elements that happen to be identical for $u_{i}$ and $u_{j}$ :

$$
\begin{aligned}
& u_{i}=(1,4,1,2,2,4) ; u_{j}=(1,2,1,3,2,4) \\
& u_{i^{\prime}}=(1,4,1,2) ; u_{j^{\prime}}=(1,2,1,3)
\end{aligned}
$$

IUUP implies that $i$ is deemed better off than $j$ if and only if $i^{\prime}$ is deemed better off than $j^{\prime}$.

IUUP stipulates that the ranks of two given individuals remain unchanged if periods in which they enjoy the same level of utilities are ignored. This condition implies that the individually representative utility functions must be additively separable.

Lemma 1. The individual strong Pareto, temporal anonymity and continuity axioms along with the independence of the utility of unconcerned periods imply that, for all $T_{i}$, the EDEU function $\Upsilon^{T_{i}}$ is additively separable and can be written as:

$$
\Upsilon^{T_{i}}\left(\left\{u_{i, s_{i}+t}\right\}_{1 \leq t \leq T_{i}}\right)=g^{-1}\left(\frac{1}{T_{i}} \sum_{t=1}^{T_{i}} g\left(u_{i, s_{i}+t}\right)\right)
$$

where $g: \Re \longmapsto \Re$ is continuous and increasing and $g(0)=0$.

We use this lemma to characterize the critical-level lifetime utility function as follows. 
Theorem 1. The ordering $R_{I}$ satisfies individual strong Pareto, temporal anonymity, continuity and IUUP if and only if, for all individuals $i$ and $j$ with utility vectors $\left\{u_{i, s_{i}+t}\right\}_{1 \leq t \leq T_{i}}$, $\left\{u_{j, s_{j}+t}\right\}_{1 \leq t \leq T_{j}} \in \mathcal{U} \backslash\left\{u_{\varnothing}\right\}$,

$$
i R_{I} j \Leftrightarrow \sum_{t=1}^{T_{i}}\left[g\left(u_{i, s_{i}+t}\right)-g(\alpha)\right] \geq \sum_{t=1}^{T_{j}}\left[g\left(u_{j, s_{j}+t}\right)-g(\alpha)\right],
$$

where $f: \Re \longmapsto \Re$ is continuous and increasing, $g(0)=0$ and $\alpha \in \Re$. If $\left\{u_{i, s_{i}+t}\right\}_{1 \leq t \leq T_{i}}$ or $\left\{u_{j, s_{j}+t}\right\}_{1 \leq t \leq T_{j}}$ is $u_{\varnothing}$, the relevant element in the summation series in (9) is replaced by zero.

This means that, in a non-discounted framework, the ordering $R_{I}$ satisfies individual strong Pareto, continuity and IUUP if and only if it can be represented by the following critical-level lifetime utility function:

$$
V_{\alpha}\left(u_{i}\right)=\sum_{t \in \mathcal{T}_{i}}\left[g\left(u_{i t}\right)-g(\alpha)\right],
$$

where $g: \Re \longmapsto \Re$ is continuous and increasing, $g(0)=0$ and $\alpha \in \Re$. Lifetime utility is thus a sum of transformed periodic utilities net of the periodic critical level, and an increase in life duration increases $V$ if and only if the new periodic values of $u_{i t}$ are greater than $\alpha$.

\subsubsection{A periodic CLGU social evaluation function}

The next step is to define a social evaluation function that obeys principles for aggregating the lifetime welfare obtained in (10). Since lifetime welfare is a function of a critical level, we now have something that can be considered as a value function (the simple sum of lifetime welfare) without being subject to the repugnant conclusion at the population level. To aggregate individual lifetime welfare, we assume that the social ordering $R$ ranking lifetime utility vectors satisfies the usual welfarist axioms characterizing classical utilitarianism: the strong Pareto, anonymity, continuity and independence of the utility of unconcerned individuals axioms.

Corollary 1. In a non-discounted framework where lifetime utilities are computed using periodic critical levels, the social ordering $R$ satisfies Classical Utilitarianism (the strong Pareto and anonymity axioms and the independence of the utilities of unconcerned individuals) if and only if, for all utility matrices $\bar{M}, \widehat{M} \in \mathcal{M} \backslash\left\{M_{\varnothing}\right\}$,

$$
\bar{M} R \widehat{M} \Leftrightarrow \sum_{i \in \overline{\mathcal{N}}_{t \in \overline{\mathcal{T}}_{i}}}\left[g\left(u_{i t}\right)-g(\alpha)\right] \geq \sum_{i \in \widehat{\mathcal{N}}} \sum_{t \in \widehat{\mathcal{T}}_{i}}\left[g\left(u_{i t}\right)-g(\alpha)\right]
$$

where $g: \Re \longmapsto \Re$ is continuous and increasing, $g(0)=0, \alpha \in \Re$, and, if $\bar{M}$ or $\widehat{M}$ is $M_{\varnothing}$, the appropriate element in the sum (11) is replaced with zero. 
Proof. The result follows directly from Theorem (1) and the characterization of generalized utilitarianism given in Theorem 5 (pp. 561-562) of Blackorby et al. (2002).

Note that if we wish to incorporate inequality aversion in the distribution of utilities (temporal and individual), the function $g$ can be chosen to be concave.

\section{Intertemporal social evaluation in a discounting frame- work}

This section explores the discounted version of the social evaluation functions developed previously. To do so, we first present the two birthdate-dependent social evaluation functions developed in the literature and propose an alternative date-dependent value function, which escapes the temporal repugnant conclusion, satisfies critical-level temporal consistency and exhibits an indifference towards fragmentation of lives.

Discounting in intertemporal social evaluation procedures is a source of controversy. The three main procedures are based on positive, negative and zero discounting. Positive discounting is commonly practiced in modern macroeconomics and in most of decisionmaking theories analyzing agents' intertemporal behaviour. Positive discounting gives utilities increasingly low weights over time; it relies largely on the assumption that, ceteris paribus, agents prefer to enjoy utilities today rather than to delay them for tomorrow, thus displaying a preference for the present.

Some advocate a totally opposite view and apply negative discounting (see for instance Loewenstein and Prelec, 2000a,b), arguing for example that agents prefer a trend of increasing living standards and a "good" end, rather than decreasing living standards and a "bad" end. This negative discounting view is reminiscent of the popular saying that "All is well that ends well": as long as one's life experience has a good end, it must be deemed as good when taken as a whole.

Between these two opposite views is the view that social discounting (positive or negative) necessitates non-welfarist information (such as birthdates and lengths of life), and is therefore inconsistent with welfarism (see for instance Blackorby et al., 1995, 1997a,b; Cowen and Parfit, 1992). In this absence of consensus, we now generalize the above framework to allow for different views on the importance of discounting.

\subsection{Principles of intertemporal social evaluation in a discounting frame- work}

\section{$3.2 \quad$ Date-adjusted periodic utilities}

To take into account possible time preferences, we define date-adjusted utilities, namely, utilities net of possible time effects. 
Definition 10. (Date-adjusted periodic utilities)

For an individual $i$ with utility vector $\left(\left\{u_{i, s_{i}+t}\right\}_{1 \leq t \leq T_{i}}\right)$, date-adjusted periodic utilities $\left(\left\{u_{i, s_{i}+t}^{0}\right\}_{1 \leq t \leq T_{i}}\right)$ are periodic utilities purged of possible time effects:

$$
u_{i, s_{i}+t}^{0}=\phi_{s_{i}+t}\left(u_{i, s_{i}+t}\right)
$$

where, for all $l \in \mathcal{Z}_{+}, \phi_{l}:$ is a bijection of $\Re \longmapsto \Re$.

The next goal is to characterize $\phi_{l}$ for all $l \in \mathcal{Z}_{+}$.

\section{Definition 11. (Date-adjusted individual strong Pareto)}

Let any two individuals $i$ and $j$ respectively have $\left(\left\{u_{i, s_{i}+t}^{0}\right\}_{1 \leq t \leq T_{i}}\right)$ and $\left(\left\{u_{j, s_{j}+t}^{0}\right\}_{1 \leq t \leq T_{j}}\right)$ as date-adjusted utility vectors, where $s_{i}$ and $s_{j}$ are respectively the birthdates of $i$ and $j$ and $T_{i}=T_{j}$ are their lengths of life.

1. If, for all $t \in\left\{1,2, \ldots, T_{i}\right\}, u_{i, s_{i}+t}^{0}=u_{j, s_{j}+t}^{0}$, then $i$ and $j$ are equally well off.

2. If, for all $t \in\left\{1,2, \ldots, T_{i}\right\}, u_{i, s_{i}+t}^{0} \geq u_{j, s_{j}+t}^{0}$, with at least one strict inequality, then individual $i$ is better off than individual $j$.

The date-adjusted individual strong Pareto axiom implies date-adjusted individual Pareto indifference, which is defined above in 1 in definition 11. Date-adjusted individual Pareto indifference requires the individual ranking to depend only on the date-adjusted periodic utilities of individuals. If the date-adjusted periodic utilities of two persons are equal, then their lifetime welfare must be the same.

\section{Definition 12. (Date-adjusted anonymity)}

Consider an individual $i$ having a date-adjusted utility vector $\left(\left\{u_{i, t}^{0}\right\}_{t \in \mathcal{T}_{i}}\right)$, and another individual $j$ whose date-adjusted utility vector can be obtained by a permutation of elements of $\left(\left\{u_{i, t}^{0}\right\}_{t \in \mathcal{T}_{i}}\right)$. The individual ordering $R_{I}$ satisfies date-adjusted anonymity if it ranks $i$ and $j$ as equally well off.

The discounted social evaluation function denoted by $V_{d}$ is defined on the set of periodic utility vectors $\mathcal{U}$ and the set of dates $\mathcal{T}$ :

$$
\begin{aligned}
V_{d}: & \mathcal{U} \longrightarrow \mathbf{R} \\
& u_{i} \longrightarrow V_{d}\left(l_{i}, u_{i}\right)=V_{d}\left(\left\{l_{t}\right\}_{t \in \mathcal{T}_{i}},\left\{u_{i, t}\right\}_{t \in \mathcal{T}_{i}}\right)=V_{d}^{0}\left(\left\{u_{i, t}^{0}\right\}_{t \in \mathcal{T}_{i}}\right) .
\end{aligned}
$$

This can be transformed to compute an Equally Distributed Equivalent Utility.

Definition 13. (The date-adjusted Equally-Distributed Equivalent Utility)

Let $\left\{u_{i t}\right\}_{t \in \mathcal{T}_{i}}$ be the utility vector of person $i$. The date-adjusted individual representative utility $\bar{u}_{i}^{d}$ is defined as the level of date-adjusted utility that, if enjoyed by person $i$ at every period of his life, makes $i$ indifferent to $\left\{u_{i t}^{0}\right\}_{t \in \mathcal{T}_{i}}$, his actual date-adjusted utility vector. 
Thus, $V_{d}^{0}\left(\bar{u}_{i}^{0} \mathbf{1}_{T_{i}}\right)=V_{d}^{0}\left(\left\{u_{i, t}^{0}\right\}_{t \in \mathcal{T}_{i}}\right)$, where $\mathbf{1}_{T_{i}}$ is a vector of dimension $T_{i}$ whose elements are all equal to 1 . The continuity and increasingness of $V_{d}^{0}$ ensure the existence of the date-adjusted EDEU, defined by:

$$
\bar{u}_{i}^{0}=\Upsilon_{d}^{T_{i}}\left(\left\{u_{i, t}^{0}\right\}_{t \in \mathcal{T}_{i}}\right) .
$$

The rank of individual $i$ is completely determined by $\left(T_{i}, \bar{u}_{i}^{0}\right)$, and we obtain:

$$
V_{d}^{0}\left(\left\{u_{i, t}\right\}_{t \in \mathcal{T}}\right)=\bar{V}_{d}^{0}\left(T_{i}, \bar{u}_{i}^{0}\right)=\bar{V}_{d}^{0}\left(T_{i}, \Upsilon_{d}^{T_{i}}\left(\left\{u_{i, t}^{0}\right\}_{t \in \mathcal{T}_{i}}\right)\right)
$$

The critical-level principle implies that:

$$
\bar{V}_{d}^{0}\left(T_{i}+1, \Upsilon_{d}^{T_{i}+1}\left(\left\{u_{i, t}^{0}\right\}_{t \in \mathcal{T}}, \alpha_{s_{i}+T_{i}}^{0}\right)\right)=\bar{V}_{d}^{0}\left(T_{i}, \Upsilon_{d}^{T_{i}}\left(\left\{u_{i, t}^{0}\right\}_{t \in \mathcal{T}}\right)\right)
$$

where $\alpha_{s_{i}+T_{i}}^{0}$ is the date-adjusted periodic critical level of person $i$.

Lemma 2. The date-adjusted individual strong Pareto, date-adjusted anonymity and continuity axioms, together with IUUP, imply that, for all $T_{i}$, the discounted individual representative utility function $\Upsilon_{d}^{T_{i}}$ is additively separable and can be written as:

$$
\Upsilon_{d}^{T_{i}}\left(\left\{u_{i, s_{i}+t}\right\}_{1 \leq t \leq T_{i}}\right)=f^{-1}\left(\frac{1}{T_{i}} \sum_{t=1}^{T_{i}} h\left(s_{i}+t, u_{i, s_{i}+t}\right)\right)
$$

where $f: \Re \longmapsto \Re$ is continuous and increasing, $f(0)=0$ and $h\left(t, u_{i, t}\right)=f\left(\phi_{t}\left(u_{i, t}\right)\right)$.

From this, we characterize the discounted lifetime utility function as follows.

Theorem 2. The ordering $R_{I}$ satisfies the date-adjusted individual strong Pareto, dateadjusted anonymity, continuity and IUUP axioms if and only if, for all individuals $i$ and $j$ with utility vectors $\left\{u_{i, s_{i}+t}\right\}_{1 \leq t \leq T_{i}},\left\{u_{j, s_{j}+t}\right\}_{1 \leq t \leq T_{j}} \in \mathcal{U} \backslash\left\{u_{\varnothing}\right\}$,

$i R_{I} j \Leftrightarrow \sum_{t=1}^{T_{i}}\left[f\left(\phi_{s_{i}+t}\left(u_{i, s_{i}+t}\right)\right)-f\left(\phi_{s_{i}+t}\left(\alpha_{s_{i}+t}\right)\right)\right] \geq \sum_{t=1}^{T_{j}}\left[f\left(\phi_{s_{j}+t}\left(u_{j, s_{j}+t}\right)\right)-f\left(\phi_{s_{j}+t}\left(\alpha_{s_{j}+t}\right)\right)\right]$,

where $f: \Re \longmapsto \Re$ is continuous and increasing, $f(0)=0, \alpha_{t} \in \Re$ for all $t$, and if $\left\{u_{i, s_{i}+t}\right\}_{1 \leq t \leq T_{i}}$ or $\left\{u_{j, s_{j}+t}\right\}_{1 \leq t \leq T_{j}}$ equals $u_{\varnothing}$, the appropriate element in the sum in (19) is replaced with zero.

Theorem 2 says that, in a discounted framework, the ordering $R_{I}$ satisfies the dateadjusted individual strong Pareto, date-adjusted anonymity, continuity and IUUP axioms if and only if it can be represented by the following discounted critical-level lifetime welfare function:

$$
V_{d}\left(u_{i}\right)=V_{d}\left(\left\{u_{i t}\right\}_{t \in \mathcal{T}_{i}}\right)=\sum_{t=1}^{T_{i}}\left[f\left(\phi_{s_{i}+t}\left(u_{i, s_{i}+t}\right)\right)-f\left(\phi_{s_{i}+t}\left(\alpha_{s_{i}+t}\right)\right)\right],
$$

where $f: \Re \longmapsto \Re$ is continuous and increasing, $f(0)=0$ and $\alpha_{t} \in \Re$ for all $t$.

Lifetime utility is thus a sum of transformed periodic utilities net of the periodic critical level, and an increase in life duration increases $V_{d}$ if and only if the new time-adjusted periodic values of $u_{i t}$ are above $\alpha_{t}$. 


\subsection{Geometric discounting of periodic utilities}

In order to simplify the above to a geometric discounting form of periodic utilities, we adapt the stationarity axiom introduced by Blackorby et al. (1997a) to the individual ordering $R_{I}$. This stationarity axiom in the case of individual rankings can be defined as follows.

Definition 14. (Stationarity with respect to $R_{I}$ ) Let $i$ and $j$ be two individuals with utility vectors $\left(\left\{u_{i, s_{i}+t}\right\}_{1 \leq t \leq T_{i}}\right)$ and $\left(\left\{u_{j, s_{j}+t}\right\}_{1 \leq t \leq T_{j}}\right)$, where $s_{i}$ and $s_{j}$ are the birthdates of $i$ and $j$, and $T_{i}$ and $T_{j}$ are their lengths of life. If $i^{\prime}$ and $j^{\prime}$ are two other individuals, identical to $i$ and $j$ in all respects except that they are born $r$ periods later, then $s_{i^{\prime}}=s_{i}+r$ and $s_{j^{\prime}}=s_{j}+r$. Assume that for all $t, u_{i^{\prime}, s_{i^{\prime}}+t}=u_{i, s_{i}+t}$ and $u_{j^{\prime}, s_{j^{\prime}}+t}=u_{j, s_{j}+t}$. Then $i R_{I} j \Leftrightarrow i^{\prime} R_{I} j^{\prime}$.

Stationarity with respect to $R_{I}$ thus requires that the ranking of two individuals remains unchanged if their dates of birth are translated by the same time constant.

Theorem 3. The ordering $R_{I}$ satisfies the date-adjusted individual strong Pareto, dateadjusted temporal anonymity, continuity, IUUP and stationarity axioms if and only if, for all individuals $i$ and $j$ with utility vectors respectively equal to $\left\{u_{i, s_{i}+t}\right\}_{1 \leq t \leq T_{i}},\left\{u_{j, s_{j}+t}\right\}_{1 \leq t \leq T_{j}}$ $\in \mathcal{U} \backslash\left\{u_{\varnothing}\right\}$

$$
i R_{I} j \Leftrightarrow \sum_{t=1}^{T_{i}} \delta^{s_{i}+t}\left[g\left(u_{i, s_{i}+t}\right)-g\left(\alpha_{s_{i}+t}\right)\right] \geq \sum_{t=1}^{T_{j}} \delta^{s_{j}+t}\left[g\left(u_{j, s_{j}+t}\right)-g\left(\alpha_{s_{j}+t}\right)\right],
$$

where $g: \Re \longmapsto \Re$ is continuous and increasing, $g(0)=0, \delta \in \Re_{++}, \alpha_{t} \in \Re$ for all $t$, and, if $\left\{u_{i, s_{i}+t}\right\}_{1 \leq t \leq T_{i}}$ or $\left\{u_{j, s_{j}+t}\right\}_{1 \leq t \leq T_{j}}$ is $u_{\varnothing}$, the relevant elements in (21) are replaced with zero.

\subsection{Discounted periodic CLGU social evaluation functions}

The next step is to define a social evaluation function that aggregates the lifetime utilities obtained in the previous step. As above, we do this by assuming that the social ordering $R$ ranking all the vectors of discounted lifetime utilities satisfies the usual welfarist axioms of classical utilitarianism (strong Pareto, anonymity, continuity and independence of the utility of unconcerned individuals (IUUI)).

Corollary 2. In an individual lifetime welfare evaluation framework in which discounting is applied geometrically using periodic critical levels, the social ordering $R$ satisfies the traditional axioms of classical utilitarianism (strong Pareto, anonymity and IUUI) if and only if, for all utility matrices $\bar{M}, \widehat{M} \in \mathcal{M} \backslash\left\{M_{\varnothing}\right\}$,

$$
\bar{M} R \widehat{M} \Leftrightarrow \sum_{i \in \overline{\mathcal{N}}} \sum_{t \in \overline{\mathcal{T}}_{i}} \delta^{s_{i}+t}\left[g\left(u_{i, t}\right)-g\left(\alpha_{s_{i}+t}\right)\right] \geq \sum_{i \in \widehat{\mathcal{N}}} \sum_{t \in \widehat{\mathcal{T}}_{i}} \delta^{s_{i}+t}\left[g\left(u_{i, t}\right)-g\left(\alpha_{s_{i}+t}\right)\right],
$$


where $g: \Re \longmapsto \Re$ is continuous and increasing, $g(0)=0, \delta \in \Re_{++}$and $\alpha_{t} \in \Re$ for all $t$, and if $\bar{M}$ or $\widehat{M}$ is $M_{\varnothing}$, the appropriate sum in (22) is replaced with zero.

Proof: The result follows directly from Theorem 3 and the characterization of generalized utilitarianism given in Theorem 5 (pp. 561-562) of Blackorby et al. (2002).

\section{Conclusion}

Development processes and policies have led to important demographic and economic changes worldwide, most importantly a fall in fertility, a growth in longevity and a general growth in living standards. This has had significant impacts on what can be termed the "quality", the "quantity" and the "duration" of life. It seems reasonable that all such three aspects of human lives should be an input into the evaluation of intertemporal social welfare.

The paper derives two sets of individual and social orderings (one with discounting and the other without) that take into account these three aspects of human lives. These orderings avoid a temporal form of the repugnant conclusion through the use of strictly positive levels of periodic critical levels, $\alpha_{t}>0$. The orderings further satisfy the properties of critical-level temporal consistency and of indifference towards fragmentation of lives. Critical-level temporal consistency ensures that periodic assessments of welfare are coherent with lifetime ones. Indifference towards fragmentation of lives positions social evaluation functions at the frontier between a preference for longevity and a preference for population renewal, ceteris paribus. These, we believe, are useful properties of social welfare evaluation procedures in an intertemporal context.

In its simplest form, the paper's social welfare framework is based on the following individual ordering function (consider (10)):

$$
V_{\alpha}\left(u_{i}\right)=\sum_{t \in \mathcal{T}_{i}} g\left(u_{i t}\right)-T_{i} g(\alpha)
$$

where $g$ is a transformation of periodic utilities $u_{i t}$ and of a periodic critical level $\alpha$, where $T_{i}$ is $i$ 's longevity, and where $i$ 's lifetime function of the critical level is $T_{i} g(\alpha)$. That framework can be extended to make the contribution of the quality of life depend upon the time at which that life is observed. That can be done using different critical levels for different calendar years. In (22), this is done by using $\alpha_{s_{i}+t}$ for $\alpha$. The framework could also be generalized by using $\alpha_{s_{i}, s_{i}+t}$ instead of $\alpha_{s_{i}+t}$, which would allow for the critical level to depend both upon the time at which the life is lived and upon the year in which the person is born. (Such a type of time and birthdate discrimination is also implicit in the discounting procedures observed in (22)). If the same weight should be used on the quality of life regardless of the time at which it is lived, then there should be no discounting and the same critical level should be used across all periods. 
Most importantly perhaps, the social evaluation functions derived in this paper enable capturing the potentially complex trade-offs between longevity, population size and living standards - trade-offs that can exist when assessing development and public policies in the presence of limited resources. The paper's social evaluation functions trade off the quality of life across individuals in the traditional manner, e.g., through imposing concavity on the $g$ function. This enables incorporating inequality aversion into intertemporal social evaluation. The trade-off between quality and quantity of life arises through the summation of differences between functions $g$ of individual welfare and the same function of the critical level, $\alpha$ - as opposed to just taking the average of periodic individual welfare functions, as has been traditionally done in welfare economics. This makes it possible to prefer a larger population to a smaller population with higher average individual welfare, if the additional lives of the larger population enjoy levels of living standards that exceed the critical level. The trade-off between the quantity and the longevity of life arises through a periodic summation of differences between $g(u)$ and $g(\alpha)$, these differences making use of periodic critical levels. A population with shorter-lived individuals may then be found preferable to one with longer lives if the quantity of lives in the first population is sufficiently large to offset the welfare value of higher longevity in the second population. A greater-longevity population may alternatively be found preferable to one with higher fertility if the first population's longer-life individuals enjoy sufficiently large levels of periodic quality of life over a sufficiently longer time horizon.

\section{Appendix}

\section{A.1. Proof of Lemma 1}

Proof. For $T_{i} \geq 3$, consider two individuals $i$ and $j$ born respectively at dates $s_{i}$ and $s_{j}$ and with $T_{i}$ and $T_{j}$ as lengths of life and $\left(\left\{u_{i, s_{i}+t}\right\}_{1 \leq t \leq T_{i}}\right)$ and $\left(\left\{u_{j, s_{j}+t}\right\}_{1 \leq t \leq T_{j}}\right)$ as utility vectors. Without loss of generality, assume that $s_{i} \leq s_{j}$. Consider now two other individuals, $i^{\prime}$ and $j^{\prime}$. $i$ and $i^{\prime}$ are identical in every period, have the same birthdate, the same length of life and the same utility vector. Individual $j^{\prime}$ is identical to $j$ except that he is born at a different date $s_{j^{\prime}}=s_{i^{\prime}}=s_{i}$.

By individual strong Pareto, $i$ and $i^{\prime}$ are equally well off, and $j$ and $j^{\prime}$ are equally well off. Let $\mathcal{P}=\left\{p_{1}, p_{2}, \ldots, p_{M}\right\}$ be a subset of $\left\{1,2, \ldots, T_{i}\right\} \bigcap\left\{1,2, \ldots, T_{j}\right\}$ for which $u_{i^{\prime}, t}=u_{j^{\prime}, t}$ for all $t \in \mathcal{P}$. By temporal anonymity, periodic utilities could be ranked so as to have $\mathcal{P}=\{1,2, \ldots, M\}$. IUUP implies that the ranking of $i^{\prime}$ and $j^{\prime}$ remains unchanged

if $\left(\left\{u_{i^{\prime}, s_{i^{\prime}}+t}\right\}_{1 \leq t \leq M}\right)$ and $\left(\left\{u_{j^{\prime}, s_{j^{\prime}}+t}\right\}_{1 \leq t \leq M}\right)$ are removed from their utility vectors. For example, consider two new individuals $i^{\prime \prime}$ and $j^{\prime \prime}$ born respectively at dates $s_{i^{\prime}}+M$ and $s_{j^{\prime}}+M$ and whose utility vectors are given by $u_{i^{\prime \prime}, t}=u_{i^{\prime}, t}$ for all $t \in\left\{M+1, M+2, \ldots, T_{i^{\prime}}\right\}$ and $u_{j^{\prime \prime}, t}=u_{j^{\prime}, t}$ for all $t \in\left\{M+1, M+2, \ldots, T_{j^{\prime}}\right\}$. By IUUP, $i^{\prime} R_{I} j^{\prime} \Leftrightarrow i^{\prime \prime} R_{I} j^{\prime \prime}$ and we 
obtain $i R_{I} j \Leftrightarrow i^{\prime \prime} R_{I} j^{\prime \prime}$ and $j R_{I} i \Leftrightarrow j^{\prime \prime} R_{I} i^{\prime \prime}$, which is equivalent to

$$
\begin{gathered}
\Upsilon^{T_{i}}\left(\left\{u_{i, s_{i}+t}\right\}_{t \in \mathcal{T}_{i}}\right) \geq \Upsilon^{T_{j}}\left(\left\{u_{j, s_{j}+t}\right\}_{t \in \mathcal{T}_{j}}\right) \Leftrightarrow \\
\Upsilon^{T_{i}-M}\left(\left\{u_{i, s_{i}+t}\right\}_{t \in \mathcal{T}_{i} \backslash \mathcal{P}}\right) \geq \Upsilon^{T_{j}-M}\left(\left\{u_{j, s_{j}+t}\right\}_{t \in \mathcal{T}_{j} \backslash \mathcal{P}}\right)
\end{gathered}
$$

and

$$
\begin{gathered}
\Upsilon^{T_{j}}\left(\left\{u_{j, s_{j}+t}\right\}_{t \in \mathcal{T}_{j}}\right) \geq \Upsilon^{T_{i}}\left(\left\{u_{i, s_{i}+t}\right\}_{t \in \mathcal{T}_{i}}\right) \Leftrightarrow \\
\Upsilon^{T_{j}-M}\left(\left\{u_{j, s_{j}+t}\right\}_{t \in \mathcal{T}_{j} \backslash \mathcal{P}}\right) \geq \Upsilon^{T_{i}-M}\left(\left\{u_{i, s_{i}+t}\right\}_{t \in \mathcal{T}_{i} \backslash \mathcal{P}}\right)
\end{gathered}
$$

where $T_{i}-M=\left|\mathcal{T}_{i} \backslash \mathcal{P}\right|$ and $T_{j}-M=\left|\mathcal{T}_{j} \backslash \mathcal{P}\right|$.

Following Blackorby et al. (1995) this implies that, in $\Upsilon^{T_{i}}, \mathcal{T}_{i} \backslash \mathcal{P}$ is separable from its complement and thus that every subset of variables is separable from its complement. This implies that $\Upsilon^{T_{i}}\left(\left\{u_{i, s_{i}+t}\right\}_{1 \leq t \leq T_{i}}\right)=F^{T_{i}}\left(\frac{1}{T_{i}} \sum_{t=1}^{T_{i}} g_{T_{i}}^{t}\left(u_{i, s_{i}+t}\right)\right)$, where $F^{T_{i}}$ and $g_{T_{i}}^{t}$ are continuous and increasing. The individual strong Pareto axiom is necessary for the additive separability of $\Upsilon^{T_{i}}$. Without that requirement, we may have $\Upsilon^{T_{i}}\left(\left\{u_{i, s_{i}+t}\right\}_{1 \leq t \leq T_{i}}\right)=$ $\min \left(\left\{u_{i, s_{i}+t}\right\}_{1 \leq t \leq T_{i}}\right)$ that also satisfies IUUP. Symmetry of $\Upsilon^{T_{i}}$ implies that each $g_{T_{i}}^{t}$ can be chosen to be independent of $t$ and by normalization, we can choose $g_{T_{i}}(0)=0$. (24) and (25) imply that $g_{T_{i}}$, and therefore $F^{T_{i}}$, can be chosen to be independent of $T_{i}$. Since $\Upsilon^{T_{i}}(v, \ldots, v)=v$ for all $v \in \Re$, we obtain

$$
\Upsilon^{T_{i}}\left(\left\{u_{i, s_{i}+t}\right\}_{1 \leq t \leq T_{i}}\right)=g^{-1}\left(\frac{1}{T_{i}} \sum_{t=1}^{T_{i}} g\left(u_{i, s_{i}+t}\right)\right),
$$

where $g: \Re \longmapsto \Re$ is continuous and increasing and $g(0)=0$.

To complete the proof, the case of $T_{i}=2,1$ and $i_{\varnothing}$ can be checked by applying the periodic critical-level principle to utility vectors of dimension less than 3 .

\section{A.2. Proof of Theorem 1}

Proof. Sufficiency is easily checked. To prove necessity, we first establish the existence of periodic critical-level functions $\left\{\alpha_{s_{i}+T_{i}} \mid s_{i}+T_{i} \in \mathcal{N}_{++}\right\}$, and show that, in a non-discounted framework, for all $i \in \mathcal{I}$ and all $\left(s_{i}, T_{i}\right) \in \mathcal{N}_{++}^{2}$, and all $\left\{u_{i, s_{i}+t}\right\}_{1 \leq t \leq T_{i}} \in \Re^{T_{i}}$,

$$
\alpha_{s_{i}+T_{i}}\left(\left\{u_{i, s_{i}+t}\right\}_{1 \leq t \leq T_{i}}\right)=\alpha_{\varnothing}=\alpha
$$

To do this, consider an individual $i$ with utility vector $\left\{u_{i, s_{i}+t}\right\}_{1 \leq t \leq T_{i}}$ and let $i^{\prime}$ be another individual identical to $i$ except that he lives for an additional period $T_{i}+1$ with utility $u_{i^{\prime}, s_{i}+T_{i}+1}=\alpha$. If the original utility vector $\left\{u_{i, s_{i}+t}\right\}_{1 \leq t \leq T_{i}}$ is removed from $u_{i}$ and $u_{i^{\prime}}$, this leads respectively to $u_{\varnothing}$ and $u_{\alpha}=\{\alpha\}$. Let $i_{\varnothing}$ be a non-existing individual enjoying 
utility $u_{\varnothing}$ and let $i_{\alpha}$ be an individual living one period (at an arbitrary date $\left.t\right)^{2}$ with utility $\alpha$.

IUUP implies that $i R_{I} i^{\prime} \Leftrightarrow i_{\varnothing} R_{I} i_{\alpha}$ and $i^{\prime} R_{I} i \Leftrightarrow i_{\alpha} R_{I} i_{\varnothing}$. Since $i_{\varnothing} I i_{\alpha}$, we have $i I i^{\prime}$, meaning that $\alpha$ is the periodic critical level for $i$. We conclude that $\alpha_{s_{i}+T_{i}}$ exists for all $i \in \mathcal{I}$ and all $\left(s_{i}, T_{i}\right) \in \mathcal{Z}_{++}^{2}$, and (27) is satisfied for all $i \in \mathcal{I}$ and all $T_{i} \in \mathcal{Z}_{++}$, and all $\left\{u_{i, s_{i}+t}\right\}_{1 \leq t \leq T_{i}} \in \Re^{T_{i}}$.

Now consider two individuals $i$ and $j$, respectively born at dates $s_{i}$ and $s_{j}$ with lengths of lives $T_{i}$ and $T_{j}$ and utility vectors $\left(\left\{u_{i, s_{i}+t}\right\}_{1 \leq t \leq T_{i}}\right)$ and $\left(\left\{u_{j, s_{j}+t}\right\}_{1 \leq t \leq T_{j}}\right)$. Without loss of generality, assume that $T_{i} \geq T_{j} \geq 3$. Let $j^{\prime}$ be an individual that is identical to $j$, except that $j^{\prime}$ lives $T_{j}^{\prime}=T_{i}$ periods and enjoys a utility of $\alpha$ during each of the additional $\left(T_{i}-T_{j}\right)$ periods. Because $\alpha$ is the periodic critical-level, $j$ and $j^{\prime}$ are equally well off, and we obtain $i R_{I} j \Leftrightarrow i R_{I} j^{\prime}$ and $j R_{I} i \Leftrightarrow j^{\prime} R_{I} i$.

Applying lemma 1, we obtain:

$$
\begin{aligned}
i R_{I} j & \Leftrightarrow i R_{I} j^{\prime} \\
& \Leftrightarrow f^{-1}\left(\frac{1}{T_{i}} \sum_{t=1}^{T_{i}} g\left(u_{i, s_{i}+t}\right)\right) \geq f^{-1}\left(\frac{1}{T_{i}}\left[\sum_{t=1}^{T_{j}} g\left(u_{j, s_{j}+t}\right)+\left(T_{i}-T_{j}\right) g(\alpha)\right]\right) \\
& \Leftrightarrow \sum_{t=1}^{T_{i}} g\left(u_{i, s_{i}+t}\right) \geq \sum_{t=1}^{T_{j}} g\left(u_{j, s_{j}+t}\right)+\left(T_{i}-T_{j}\right) g(\alpha) \\
& \Leftrightarrow \sum_{t=1}^{T_{i}}\left[g\left(u_{i, s_{i}+t}\right)-g(\alpha)\right] \geq \sum_{t=1}^{T_{j}}\left[g\left(u_{j, s_{j}+t}\right)-g(\alpha)\right]
\end{aligned}
$$

and

$$
j R_{I} i \Leftrightarrow \sum_{t=1}^{T_{j}}\left[g\left(u_{j, s_{j}+t}\right)-g(\alpha)\right] \geq \sum_{t=1}^{T_{i}}\left[g\left(u_{i, s_{i}+t}\right)-g(\alpha)\right]
$$

which implies (9).

\section{A.3. Proof of Lemma 2}

Proof. For $T_{i} \geq 3$, consider two individuals $i$ and $j$ born on dates $s_{i}$ and $s_{j}$ with lengths of life $T_{i}$ and $T_{j}$ and with utility vectors $\left(\left\{u_{i, s_{i}+t}\right\}_{1 \leq t \leq T_{i}}\right)$ and $\left(\left\{u_{j, s_{j}+t}\right\}_{1 \leq t \leq T_{j}}\right)$. Their date-adjusted utility vectors are $\left(\left\{u_{i, s_{i}+t}^{0}\right\}_{1 \leq t \leq T_{i}}\right)$ and $\left(\left\{u_{j, s_{j}+t}^{0}\right\}_{1 \leq t \leq T_{j}}\right)$. Without loss of generality, assume that $s_{i} \leq s_{j}$.

Let $i^{\prime}$ and $j^{\prime}$, two other individuals born on date 0 and with respective date-adjusted utility vectors $\left(\left\{u_{i^{\prime}, s_{i^{\prime}}+t}^{0}\right\}_{1 \leq t \leq T_{i^{\prime}}}\right)=\left(\left\{u_{i, s_{i}+t}^{0}\right\}_{1 \leq t \leq T_{i}}\right)=\left(\left\{\phi_{s_{i}+t}\left(u_{i, s_{i}+t}\right)\right\}_{1 \leq t \leq T_{i}}\right)$ and $\left(\left\{u_{j^{\prime}, s_{j^{\prime}}+t}\right\}_{1 \leq t \leq T_{j^{\prime}}}\right)=$ $\left(\left\{u_{j, s_{j}+t}^{0}\right\}_{1 \leq t \leq T_{j}}\right)=\left(\left\{\phi_{s_{j}+t}\left(u_{j, s_{j}+t}\right)\right\}_{1 \leq t \leq T_{j}}\right)$. The date-adjusted individual strong Pareto axiom means that $i$ and $i^{\prime}$ are equally well off, as are $j$ and $j^{\prime}$.

\footnotetext{
${ }^{2}$ The date does not matter here since we are working in a non-discounted framework.
} 
Let $\mathcal{P}=\left\{p_{1}, p_{2}, \ldots, p_{M}\right\}$ be a subset of $\left\{1,2, \ldots, T_{i}\right\} \bigcap\left\{1,2, \ldots, T_{j}\right\}$ for which $u_{i^{\prime}, s_{i^{\prime}}+t}^{0}=$ $u_{j^{\prime}, s_{j^{\prime}}+t}^{0}$ for all $t \in \mathcal{P}$. The date-adjusted anonymity axiom allows date-adjusted periodic utilities to be ranked to have $\mathcal{P}=\{1,2, \ldots, M\}$. Given that $i^{\prime}$ and $j^{\prime}$ have the same date of birth (date 0), IUUP and given the bijectivity of $\phi_{l}$, the ranking of $i^{\prime}$ and $j^{\prime}$ remains unchanged if $\left(\left\{u_{i^{\prime}, s_{i^{\prime}}+t}\right\}_{1 \leq t \leq M}\right)$ and $\left(\left\{u_{j^{\prime}, s_{j^{\prime}}+t}\right\}_{1 \leq t \leq M}\right)$ are removed from their utility vectors.

Consider then two new individuals $i^{\prime \prime}$ and $j^{\prime \prime}$ born at date $M$ and whose date-adjusted utility vectors are given by $u_{i^{\prime \prime}, t}^{0}=u_{i^{\prime}, t}^{0}$ for all $t \in\left\{M+1, M+2, \ldots, T_{i^{\prime}}\right\}$ and $u_{j^{\prime \prime}, t}^{0}=u_{j^{\prime}, t}^{0}$ for all $t \in\left\{M+1, M+2, \ldots, T_{j^{\prime}}\right\}$. By IUUP and the bijectivity of $\phi_{l}, i^{\prime} R_{I} j^{\prime} \Leftrightarrow i^{\prime \prime} R_{I} j^{\prime \prime}$, and we find $i R_{I} j \Leftrightarrow i^{\prime \prime} R_{I} j^{\prime \prime}$ and $j R_{I} i \Leftrightarrow j^{\prime \prime} R_{I} i^{\prime \prime}$, which is equivalent to:

$$
\begin{gathered}
\Upsilon_{d}^{T_{i}}\left(\left\{u_{i, s_{i}+t}^{0}\right\}_{t \in \mathcal{T}_{i}}\right) \geq \Upsilon_{d}^{T_{j}}\left(\left\{u_{j, s_{j}+t}^{0}\right\}_{t \in \mathcal{T}_{j}}\right) \Leftrightarrow \\
\Upsilon_{d}^{T_{i}-M}\left(\left\{u_{i, s_{i}+t}^{0}\right\}_{t \in \mathcal{T}_{i} \backslash \mathcal{P}}\right) \geq \Upsilon^{T_{j}-M}\left(\left\{u_{j, s_{j}+t}^{0}\right\}_{t \in \mathcal{T}_{j} \backslash \mathcal{P}}\right)
\end{gathered}
$$

and

$$
\begin{gathered}
\Upsilon_{d}^{T_{j}}\left(\left\{u_{j, s_{j}+t}^{0}\right\}_{t \in \mathcal{T}_{j}}\right) \geq \Upsilon_{d}^{T_{i}}\left(\left\{u_{i, s_{i}+t}^{0}\right\}_{t \in \mathcal{T}_{i}}\right) \Leftrightarrow \\
\Upsilon^{T_{j}-M}\left(\left\{u_{j, s_{j}+t}^{0}\right\}_{t \in \mathcal{T}_{j} \backslash \mathcal{P}}\right) \geq \Upsilon_{d}^{T_{i}-M}\left(\left\{u_{i, s_{i}+t}^{0}\right\}_{t \in \mathcal{T}_{i} \backslash \mathcal{P}}\right),
\end{gathered}
$$

where $T_{i}-M=\left|\mathcal{T}_{i} \backslash \mathcal{P}\right|$ and $T_{j}-M=\left|\mathcal{T}_{j} \backslash \mathcal{P}\right|$.

Following the proof of lemma $1, \mathcal{T}_{i} \backslash \mathcal{P}$ in $\Upsilon_{d}^{T_{i}}$ is separable from its complement. Therefore, every subset of variables is separable from its complement, and this implies that:

$$
\Upsilon_{d}^{T_{i}}\left(\left\{u_{i, s_{i}+t}^{0}\right\}_{1 \leq t \leq T_{i}}\right)=F^{T_{i}}\left(\frac{1}{T_{i}} \sum_{t=1}^{T_{i}} f_{T_{i}}^{t}\left(u_{i, s_{i}+t}^{0}\right)\right),
$$

where $F^{T_{i}}$ and $f_{T_{i}}^{t}$ are continuous and increasing. The date-adjusted individual strong Pareto axiom is necessary for additive separability of $\Upsilon_{d}^{T_{i}}$. Without this requirement, we could have $\Upsilon_{d}^{T_{i}}\left(\left\{u_{i, s_{i}+t}^{0}\right\}_{1 \leq t \leq T_{i}}\right)=\min \left(\left\{u_{i, s_{i}+t}^{0}\right\}_{1 \leq t \leq T_{i}}\right)$, which also satisfies the IUUP axiom. Symmetry of $\Upsilon_{d}^{T_{i}}$ with respect to the date-adjusted periodic utilities implies that each $f_{T_{i}}^{t}$ can be chosen to be independent of $t$ and, normalizing, we can choose $f_{T_{i}}(0)=0$. (33) and (34) imply that $f_{T_{i}}$, and therefore $F^{T_{i}}$, can be chosen to be independent of $T_{i}$. Since $\Upsilon_{d}^{T_{i}}(v, \ldots, v)=v$ for all $v \in \Re$, we obtain:

$$
\Upsilon_{d}^{T_{i}}\left(\left\{u_{i, s_{i}+t}^{0}\right\}_{1 \leq t \leq T_{i}}\right)=f^{-1}\left(\frac{1}{T_{i}} \sum_{t=1}^{T_{i}} f\left(u_{i, s_{i}+t}^{0}\right)\right)
$$

where $f: \Re \longmapsto \Re$ is continuous and increasing, $f(0)=0$.

Substituting $\phi_{s_{i}+t}\left(u_{i, s_{i}+t}\right)$ in (35) for $u_{i, s_{i}+t}^{0}$, we obtain

$$
\Upsilon_{d}^{T_{i}}\left(\left\{u_{i, s_{i}+t}\right\}_{1 \leq t \leq T_{i}}\right)=f^{-1}\left(\frac{1}{T_{i}} \sum_{t=1}^{T_{i}} h\left(s_{i}+t, u_{i, s_{i}+t}\right)\right),
$$


where $f: \Re \longmapsto \Re$ is continuous and increasing, $f(0)=0$, and $h\left(t, u_{i, t}\right)=f\left(\phi_{t}\left(u_{i, t}\right)\right)$.

The proof can be extended to the case of $T_{i}=2,1$ and $i_{\varnothing}$ by applying the periodic critical-level principle to utility vectors of dimension less than 3 .

\section{A.4. Proof of Theorem 2}

Proof. Sufficiency is easily checked. To prove necessity, consider two individuals $i$ and $j$ born on dates $s_{i}$ and $s_{j}$, with lengths of life $T_{i}$ and $T_{j}$, and with utility vectors $\left(\left\{u_{i, s_{i}+t}\right\}_{1 \leq t \leq T_{i}}\right)$ and $\left(\left\{u_{j, s_{j}+t}\right\}_{1 \leq t \leq T_{j}}\right)$. Without loss of generality, assume that $T_{i} \geq T_{j} \geq 3$. Let $j^{\prime}$ be an individual identical to $j$ except that he lives $T_{j^{\prime}}=T_{i}$ periods and in every additional period enjoys the corresponding periodic critical-level utility. Thus, $s_{j^{\prime}}=s_{j}, u_{j^{\prime}, s_{j^{\prime}}+t}=u_{j, s_{j}+t}$ for $t \leq T_{j}$ and $u_{j^{\prime}, s_{j^{\prime}}+t}=\alpha_{s_{j}+t}$ for $t>T_{j}$. Since $\alpha_{t}$ is the period $t$ critical-level utility, $j$ and $j^{\prime}$ are equally well off, and we find that: $i R_{I} j \Leftrightarrow i R_{I} j^{\prime}$ and $j R_{I} i \Leftrightarrow j^{\prime} R_{I} i$.

Applying lemma 2, we obtain:

$$
\begin{aligned}
i R_{I} j \quad \Leftrightarrow \quad i R_{I} j^{\prime} & \Leftrightarrow \quad f^{-1}\left(\frac{1}{T_{i}} \sum_{t=1}^{T_{i}} f\left(\phi_{s_{i}+t}\left(u_{i, s_{i}+t}\right)\right)\right) \geq \\
& f^{-1}\left(\frac{1}{T_{i}}\left[\sum_{t=1}^{T_{j}} f\left(\phi_{s_{j}+t}\left(u_{j, s_{j}+t}\right)\right)+\sum_{t=T_{j}+1}^{T_{i}} f\left(\phi_{s_{j}+t}\left(\alpha_{s_{j}+t}\right)\right)\right]\right) \\
\Leftrightarrow \quad & \sum_{t=1}^{T_{i}} f\left(\phi_{s_{i}+t}\left(u_{i, s_{i}+t}\right)\right)-\sum_{t=1}^{T_{j}} f\left(\phi_{s_{j}+t}\left(\alpha_{s_{j}+t}\right)\right) \geq \\
& \sum_{t=1}^{T_{j}} f\left(u_{j, s_{j}+t}\right)+\sum_{t=T_{j}+1}^{T_{i}} f\left(\phi_{s_{j}+t}\left(\alpha_{s_{j}+t}\right)\right)-\sum_{t=1}^{T_{j}} f\left(\phi_{s_{j}+t}\left(\alpha_{s_{j}+t}\right)\right) \\
\Leftrightarrow \quad & \sum_{t=1}^{T_{i}}\left[f\left(\phi_{s_{i}+t}\left(u_{i, s_{i}+t}\right)\right)-f\left(\phi_{s_{j}+t}\left(\alpha_{s_{j}+t}\right)\right)\right] \geq \\
& \sum_{t=1}^{T_{j}}\left[f\left(\phi\left(\left(u_{j, s_{j}+t}\right)\right)-f\left(\phi_{s_{j}+t}\left(\alpha_{s_{j}+t}\right)\right)\right] .\right.
\end{aligned}
$$

Since $\phi_{s_{i}+t}\left(\alpha_{s_{i}+t}\right)=\alpha_{0}=\phi_{s_{j}+t}\left(\alpha_{s_{j}+t}\right)$, we obtain:

$$
\begin{array}{r}
i R_{I} j \Leftrightarrow \sum_{t=1}^{T_{i}}\left[f\left(\phi_{s_{i}+t}\left(u_{i, s_{i}+t}\right)\right)-f\left(\phi_{s_{i}+t}\left(\alpha_{s_{i}+t}\right)\right)\right] \geq \\
\sum_{t=1}^{T_{j}}\left[f\left(\phi\left(\left(u_{j, s_{j}+t}\right)\right)-f\left(\phi_{s_{j}+t}\left(\alpha_{s_{j}+t}\right)\right)\right],\right.
\end{array}
$$

and, similarly, that:

$$
j R_{I} i \Leftrightarrow \sum_{t=1}^{T_{j}}\left[f\left(\phi\left(u_{j, s_{j}+t}\right)\right)-f\left(\phi_{s_{j}+t}\left(\alpha_{s_{j}+t}\right)\right)\right] \geq \sum_{t=1}^{T_{i}}\left[f\left(\phi_{s_{i}+t}\left(u_{i, s_{i}+t}\right)\right)-f\left(\phi_{s_{i}+t}\left(\alpha_{s_{i}+t}\right)\right)\right]
$$


which implies (19).

\section{A.5. Proof of Theorem 3}

Proof. Sufficiency is easily verified. To prove necessity, let $g(u)=h(0, u)=f\left(\phi_{0}(u)\right)$, where $h, f$ and $\phi_{0}$ are as in the statement of lemma 2 and theorem 2. We must show that:

$$
f\left(\phi_{s_{i}+t}\left(u_{i, s_{i}+t}\right)\right)-f\left(\phi_{s_{i}+t}\left(\alpha_{s_{i}+t}\right)\right)=\delta^{s_{i}+t}\left[g\left(u_{i, s_{i}+t}\right)-g\left(\alpha_{s_{i}+t}\right)\right]
$$

for all $\left(s_{i}, t, u_{i, s_{i}+t}\right) \in \mathcal{Z}_{+}^{2} \times \Re$, where $\delta \in \Re_{++}$.

Consider the sets $\mathcal{I}_{t}$, the sets of all individuals $i$ born at date $t$. Stationarity implies that the individual ordering induced in these sets are the same for all $t$ because the utility profile of all the individuals in $\mathcal{I}_{t}$ can be obtained from individuals in $\mathcal{I}_{0}$ by adding $r=t$ periods to the birthdate of each person.

Now consider an individual $i$ born on date $t$ and living 2 periods with periodic utilities $u_{i, t}$ and $u_{i, t+1}$ which, together with the above remark, implies that

$$
h\left(t+r, u_{i, t+r}\right)+h\left(t+r+1, u_{i, t+r+1}\right)=F_{r}\left(h\left(t, u_{i, t}\right)+h\left(t+1, u_{i, t+1}\right)\right)
$$

where $h\left(t, u_{i, t}\right)=f\left(\phi_{t}\left(u_{i, t}\right)\right)$ for all $t \in \mathcal{Z}_{+}$. Note that $F_{r}$ does not depend on $t$ because the ordering of $\mathcal{I}_{t}$ is the same for all $t$.

Define

$$
\bar{h}(t+r, z)=h\left(t+r, g_{t}^{-1}(z)\right)=h(t+r, u), x=h\left(t, u_{i, t}\right), y=h\left(t+1, u_{i, t+1}\right)
$$

where $g_{t}()=.h(t,$.$) .$

The following functional equation is then obtained:

$$
\bar{h}(t+r, x)+\bar{h}(t+1+r, y)=F_{r}(x+y)
$$

This is a Pexider equation (further details in Aczél 1966 and Blackorby et al. 1997a). Its solution is given by

$$
\bar{h}(t+r, z)=a(r) z+b(r),
$$

where $a$ and $b$ cannot depend on $t$ because $F_{r}$ does not and $z$ is an independent variable. Therefore,

$$
h(t+r, u)=a(r) h(t, u)+b(r) .
$$

Setting $t=0$, we find that:

$$
h(r, u)=a(r) g(u)+b(r) .
$$

Thus,

$$
h(t+r, u)=a(t+r) g(u)+b(t+r)
$$


Futhermore, setting $r=0$ in (54), it follows that:

$$
h(0, u)=g(u)=a(0) g(u)+b(0) .
$$

Because $g(0)=0$, this implies that $a(0)=1$ and $b(0)=0$. Substituting (54) into (53), we obtain:

$$
h(t+r, u)=a(r)[a(t) g(u)+b(t)]+b(r) .
$$

Combining (55) and (57), it follows that:

$$
a(t+r) g(u)+b(t+r)=a(r)[a(t) g(u)+b(t)]+b(r),
$$

which is equivalent to

$$
[a(t+r)-a(r) a(t)] g(u)=a(r) b(t)+b(r)-b(t+r) .
$$

Because $g$ is increasing and the right-hand side of (59) is independent of $u$, this requires that

$$
a(t+r)-a(r) a(t)=0
$$

and that

$$
a(r) b(t)+b(r)-b(t+r)=0 .
$$

Thus, $a(t+r)=a(r) a(t)$ and $b(t+r)=a(r) b(t)+b(r)$ for all $t, r \in \mathcal{Z}_{++}$, Denoting $a(1)=\delta$, we obtain $a(2)=\delta^{2}, a(t)=\delta^{t}$, and $a(t+1)=\delta^{t+1}$. Thus, (54) becomes

$$
h(t, u)=\delta^{t} g(u)+b(t) .
$$

Substituting $u$ by $\alpha_{t}$, it follows that:

$$
h\left(t, \alpha_{t}\right)=\delta^{t} g\left(\alpha_{t}\right)+b(t)
$$

which implies that:

$$
b(t)=h\left(t, \alpha_{t}\right)-\delta^{t} g\left(\alpha_{t}\right) .
$$

Substituting in (62), it follows that

$$
h\left(s_{i}+t, u_{i, s_{i}+t}\right)=\delta^{s_{i}+t}\left[g\left(u_{i, s_{i}+t}\right)-g\left(\alpha_{s_{i}+t}\right)\right]+h\left(s_{i}+t, \alpha_{s_{i}+t}\right),
$$

or, equivalently, that

$$
f\left(\phi_{s_{i}+t}\left(u_{i, s_{i}+t}\right)\right)-f\left(\phi_{s_{i}+t}\left(\alpha_{s_{i}+t}\right)\right)=\delta^{s_{i}+t}\left[g\left(u_{i, s_{i}+t}\right)-g\left(\alpha_{s_{i}+t}\right)\right]
$$

which is (48). 


\section{References}

ACZÉL, J. (1966): Lectures on Functional Equations and Their Applications, New York: Academic Press.

Blackorby, C., W. Bossert, and D. Donaldson (1995): "Intertemporal Population Ethics: Critical-Level Utilitarian Principles," Econometrica, 63, 1303-20.

(1996a): "Intertemporally Consistent Population Ethics: Classical Utilitarian Principles," in Social Choice Re-examined, ed. by K. Arrow, A. Sen, and K. Suzumura, New York: Palgrave MacMillan, vol. 2, 137-162.

(1996b): "Quasi Ordering and Population Ethics," Social Choice and Welfare, 13, $129-150$.

(1997a): "Birth-Date Dependent Population Ethics: Critical-Level Principles," Journal of Economic Theory, 77, 260-84.

(1997b): "Intertemporally Consistent Population Ethics: Birth-Date Dependent Classical Principles," The Japanese Economic Review, 48, 267-292.

- (2002): "Utilitarianism and the theory of justice," in Handbook of Social Choice and Welfare, ed. by K. J. Arrow, A. K. Sen, and K. Suzumura, Elsevier, vol. 1 of Handbook of Social Choice and Welfare, chap. 11, 543-596.

(2005): Population issues in social choice theory, welfare economics and ethics, New York: Cambridge University Press.

Blackorby, C. and D. Donaldson (1984): "Social Criteria for Evaluating Population Change," Journal of Public Economics, 25, 13-33.

Cowen, T. And D. Parfit (1992): "Against the social discount rate," in Justice between age groups and generations, ed. by P. Laslett and J. S. Fishkin, Yale University Press: New Haven, 144-161.

Dalgaard, C.-J. And H. Strulik (2011): "Optimal Aging and Death: Understanding the Preston Curve," LEPAS-Long-Run Economic Perspectives of an Ageing Society, Second LEPAS Workshop on The Economics of Ageing.

Debreu, G. (1959): Theory of Value: An Axiomatic Analysis of Economic Equilibrium, New Haven and London: Yale University Press.

Ehrlich, I. And H. Chuma (1990): "A Model of the Demand for Longevity and the Value of Life Extension," Journal of Political Economy, 98, 761-82. 
Grossman, M. (1972): The Demand for Health: A Theoretical and Empirical Investigation, no. 72-1 in NBER Books, National Bureau of Economic Research, Inc.

Loewenstein, G. And D. Prelec (2000a): "Anomalies in Intertemporal Choice: Evidence and an Interpretation," in Choices, values, and frames, ed. by D. Kahneman and A. Tversky, Cambridge, UK: Cambridge University Press.

(2000b): "Preferences for Sequences of Outcomes," in Choices, values, and frames, ed. by D. Kahneman and A. Tversky, Cambridge, UK: Cambridge University Press.

MaAsoumi, E. And S. Zandvakili (1986): "A Class of Generalized Measures of Mobility with Applications," Economics Letters, 22, 97-102.

PARfit, D. (1984): Reasons and Persons, Oxford: Oxford University Press.

Preston, S. (1975): "The changing relation between mortality and level of economic development," Population Studies, 29, 231-248.

SAlas, R. And I. RABAdAn (1998): "Lifetime and Vertical Intertemporal Inequality, Income Smoothing, and Redistribution: A Social Welfare Approach," Review of Income and Wealth, 44, 63-79.

Schultz, P. (2003): 1,000 Places to See Before You Die: A Traveler's Life List, New York, NY, USA: Workman Publishing.

UNDP (1990): "Human Development Report 1990," Tech. rep., United Nations Development Programme, Oxford University Press. 


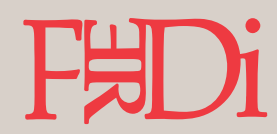

Créée en 2003, la Fondation pour les études et recherches sur le développement international vise à favoriser la compréhension du développement économique international et des politiques qui l'influencent.

\section{$\rightarrow$ Contact}

www.ferdi.fr

contact@ferdi.fr

+33(0)473177530 